\title{
Welche Fehler zeigt das Peer Review Verfahren auf?
}

Im HELIOS Peer Review Verfahren werden von 2 externen Chefärzten (Peers) retrospektiv Patientenakten einer Klinik aufgrund von auffälligen Qualitätsindikatoren untersucht. Qualitätsindikatoren sind beispielsweise Krankenhaussterblichkeiten bei bestimmten Erkrankungen, wie z.B. die Krankenhaussterblichkeit bei Herzinsuffizienz. Die Patientenakten werden von den Peers auf Verbesserungspotential untersucht und entsprechend eingestuft. Die Einstufung erfolgt in 3 Kategorien: Kategorie 1 bedeutet - Verbesserungspotential vorhanden, Kategorie 2 bedeutet - Fehlkodierung, Kategorie 3 bedeutet suffiziente Therapie. Maßstab der retrospektiven Patientenaktenanalyse und damit für das Auffinden von Verbesserungspotenzial ist die individuell optimale Patientenbehandlung unter Beachtung folgender Analysekriterien:

- Diagnostik und Behandlung adäquat und zeitgerecht?

- Behandlungsprozess zielführend und zeitnah kritisch hinterfragt?

- Indikation zur OP/Intervention/Intensivtherapie angemessen und rechtzeitig?

- Behandlungsleitlinien und Standards berücksichtigt?

- Dokumentation umfassend und schlüssig?

- Interdisziplinäre Zusammenarbeit reibungslos?

- Kontrolle der Behandlungsverläufe?

Fehler sind Zielverfehlungen einer individualisierten optimalen Patientenbehandlung trotz vorhandenem Wissen und Können [1]. Bei den Fehlern unterscheidet man aktives Abweichen vom optimalen Behandlungsprozess (aktiver Fehler) und unbewusstes Abweichen vom optimalen Behandlungsprozess (passiver Fehler). Unzureichendes Handeln aufgrund mangelnden Wissens (nach obiger strenger Definition kein Fehler) entspricht in der Regel einer unzureichenden Professionalität, begründet in einem Aus- oder Weiterbildungsdefizit [1]. Fehlertheoretisch lassen sich noch latente Bedingungen abgrenzen aufgrund von strukturellen (z.B. bauliche Gegebenheiten) oder prozessoralen Bedingungen (z.B. Dienstplangestaltung) [1]. Neben den individuellen Fehlern spielen im Klinikbereich insbesondere Teamfehler eine große Rolle. Nicht selten liegen Mischformen von Fehlern vor, Fehlerketten können zu einem negativen Ereignis führen [1].

Im Verbesserungspotenzial der HELIOS Peer Review Verfahren lassen sich alle der erwähnten Fehlerarten nachweisen. Individuelle Fehler mit bewusstem Abweichen von der individuell optimalen Therapie (direkte Fehler) sind selten. Häufiger findet man unbewusstes Abweichen von der optimalen Therapie trotz vorhandener Kompetenz (passiver Fehler) und Wissensdefizite. Da es sich um eine retrospektive Patientenaktenanalyse handelt, wird der Behandlungsprozess nur aufgrund der vorhandenen Dokumentationen beurteilt. Die Dokumentation in der Patientenakte ist teilweise unzureichend. Dies bedeutet nicht immer, dass die Behandlung nicht adäquat durchgeführt wurde, ist jedoch häufig auch Ausdruck einer nicht zielgerichteten Therapie und somit Hinweis auf ein Qualitätsdefizit. Teamfehler bestehen häufig in einer unzureichenden Kommunikation oder einer mangelhaften interdisziplinären Zusammenarbeit mit konsekutivem Behandlungsdefizit. Latente fehlerfördernde Bedingungen, wie lückenhafte strukturelle Voraussetzungen (z.B. fehlende Gerätetechnik) oder unzureichende Prozessorganisationen (unangemessene OP-Planung) sind weniger häufig.

Autorenerklärung: Der Autor hat keine finanziellen Interessenkonflikte.

\footnotetext{
Literatur

1 Hofinger G. Fehler und Ursache. In: Badke-Schaub P, Hofinger G, Lauche K (Hrsg). Human Factors. Psychologie sicheren Handelns in Risikobranchen. Heidelberg: 2008: 37-55
}

W. Krahwinkel

Qualitätsmanagement

Schlüsselwörter

Peer Review

Qualitätsindikatoren

Behandlungsverbesserung

Keywords

peer review

quality indicators

treatment improvement

Institut

HELIOS Krankenhaus Leisnig

Bibliografie

Dol 10.1055/s-0031-1286082 Dtsch Med Wochenschr 2011; 136: S53 - (c) Georg Thieme Verlag KG Stuttgart · New York . ISSN 0012-0472

Korrespondenz Dr. med. Wolfgang Krahwinkel Klinik für Innere Medizin und Intensivmedizin HELIOS Krankenhaus Leisnig Colditzer Straße 48 04703 Leisnig

Tel. 034321/8-231

Fax 034321/8-244 eMail wolfgang.krahwinkel@ helios-kliniken.de 